Bulletin of the Section of Logic

Volume 45:3/4 (2016), pp. 155-169

http://dx.doi.org/10.18778/0138-0680.45.3.4.02

Emília Halušková*

\title{
ON DIRECT LIMIT CLOSED CLASSES OF ALGEBRAS
}

\begin{abstract}
Axiomatic classes of algebras of a given type which are closed with respect to direct limits are studied in this paper.

Keywords: algebra, direct limit, closed class, term, formula, sentence, monounary algebra
\end{abstract}

\section{Introduction}

Let $\mathcal{K}$ be a class of algebras and $\underline{\mathbf{L}}(\mathcal{K})$ be the class of all direct limits of algebras of $\mathcal{K}$. If every algebra of $\overrightarrow{\underline{L}}(\mathcal{K})$ is isomorphic to an algebra of $\mathcal{K}$, then we say that $\mathcal{K}$ is an $\underline{\mathbf{L}}$-class or that $\mathcal{K}$ is closed with respect to direct limits.

Every variety is an $\underline{\mathbf{L}}$-class. Every $\underline{\mathbf{L}}$-class is closed with respect to retracts. Arbitrary intersections and finite unions of $\underline{\mathbf{L}}$-classes are $\underline{\text { L}}$-classes. The class of all algebras which have the cardinality bounded

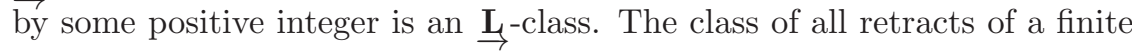
algebra is an $\underline{\mathbf{L}}$-class, cf.[3].

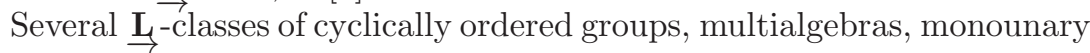
algebras resp. were studied in the papers [6], [10], [2] resp.

We deal with axiomatic $\underline{\mathbf{L}}$-classes of algebras. All axiomatic $\underline{\mathbf{L}}$-classes of the first order logic are described in Theorem 3. This description uses Theorem 6 of [8]. It is sufficient to consider direct limit systems of algebras

*2000 Mathematics Subject Classification. 08B25, 03C05, 08A60. Supported by the grant VEGA 2/0044/16. 
indexed by linearly ordered natural numbers in this case. The section 5 contains an example of an $\underline{\mathbf{L}}$-class of (monounary) algebras which is not possible to describe as an axiomatic class of the first order. Theorem 1 and Theorem 4 present elementary $\underline{\mathbf{L}}$-classes which are obtained by generalizations of the direct limit preserving sentence from Exercise 5.2.24 of [9]. Theorem 4 refers monounary algebras.

\section{Preliminaries}

The symbol $\mathbb{N}$ denotes the set of all positive integers and the symbol $\mathbb{N}_{0}$ denotes the set of all non-negative integers in this paper. We denote by Ord the class of all ordinals.

We start with the notion of a direct limit, cf. [1], §21.

Let $\langle P, \leq\rangle$ be a directed partially ordered set. For each $p \in P$, let $\mathcal{A}_{p}=\left(A_{p}, F\right)$ be an algebra of some fixed type $F$. Assume that if $p, q \in$ $P, p \neq q$, then $A_{p} \cap A_{q}=\emptyset$. Suppose that for each pair of elements $p$ and $q$ in $P$ with $p<q$, we have a homomorphism $\varphi_{p q}$ of $\mathcal{A}_{p}$ into $\mathcal{A}_{q}$ such that $p<q<s$ implies that $\varphi_{p s}=\varphi_{p q} \circ \varphi_{q s}$. For each $p \in P$, suppose that $\varphi_{p p}$ is the identity on $A_{p}$. The family $\left\{P, \mathcal{A}_{p}, \varphi_{p q}\right\}$ is said to be direct.

Assume that $p, q \in P$ and $x \in A_{p}, y \in A_{q}$. Put $x \equiv y$ if there exists $s \in P$ with $p \leq s, q \leq s$ such that $\varphi_{p s}(x)=\varphi_{q s}(y)$. For each $z \in \bigcup_{p \in P} A_{p}$ put $\bar{z}=\left\{t \in \bigcup_{p \in P} A_{p}: z \equiv t\right\}$. Denote

$$
\bar{A}=\left\{\bar{z}: z \in \bigcup_{p \in P} A_{p}\right\} .
$$

Let $f \in F$ be an $n$-ary operation. Let $x_{j} \in A_{p_{j}}, 1 \leq j \leq n$ and let $s \in P$ be an upper bound of $p_{j}$. Define $f\left(\overline{x_{1}}, \ldots, \overline{x_{n}}\right)=\overline{f\left(\varphi_{p_{1} s}\left(x_{1}\right), \ldots, \varphi_{p_{n} s}\left(x_{n}\right)\right)}$. Then the algebra $\overline{\mathcal{A}}=(\bar{A}, F)$ is said to be a direct limit of the direct family $\left\{P, \mathcal{A}_{p}, \varphi_{p q}\right\}$. We express this situation as follows

$$
\left\{P, \mathcal{A}_{p}, \varphi_{p q}\right\} \longrightarrow \overline{\mathcal{A}}
$$

The definition yields the following statement.

Lemma 1. Let $\mathcal{A}$ be an algebra. If (1) is valid, $\mathcal{A}_{p} \cong \mathcal{A}$ and $\varphi_{p q}$ is an isomorphism from $\mathcal{A}_{p}$ into $\mathcal{A}_{q}$, then $\overline{\mathcal{A}} \cong \mathcal{A}$.

Let $\mathcal{K}$ be a nonempty class of algebras of type $F$. Then $\mathbf{I}(\mathcal{K})$ denotes the class of all isomorphic copies of algebras of $\mathcal{K}$, 
$\underline{\mathbf{L}}(\mathcal{K})$ denotes the class of all direct limits of algebras of $\mathcal{K}$, if $\mathbf{I}(\underline{\mathbf{L}}(\mathcal{K}))=\mathbf{I}(\mathcal{K})$, then we say that $\mathcal{K}$ is an $\underline{\mathbf{L}}$-class. The empty class is an $\underline{\mathbf{L}}$-class.

We denote by $T_{F}$ the set of all terms of type $F$. That means that $T_{F}$ is the least possible set such that $T_{F}$ contains all projections, $F \subseteq T_{F}$ and $T_{F}$ is closed under compositions, cf. e.g. [5].

The following fact will be used in the proof of Lemma 4.

Lemma 2. Let (1) be valid and $f, g \in T_{F}$ be $n$-ary, $n \in \mathbb{N}$.

If $u_{1}, \ldots, u_{n} \in \bar{A}$ are such that

$$
f\left(u_{1}, \ldots, u_{n}\right)=g\left(u_{1}, \ldots, u_{n}\right),
$$

then there are $p \in P$ and $a_{1}, \ldots, a_{n} \in A_{p}$ such that

$$
f\left(a_{1}, \ldots, a_{n}\right)=g\left(a_{1}, \ldots, a_{n}\right) \text { and } a_{1} \in u_{1}, \ldots, a_{n} \in u_{n} .
$$

\section{Sentences preserved by direct limits}

We can look on algebras of type $F$ as on structures of the model theory, cf. e.g.[1].

We have that $F$ is a signature. We will use the notation introduced in [4], §2.1. Let $F_{\omega \omega}, F_{\infty \omega}, F_{\omega 0}, F_{\infty 0}, F_{\infty \infty}$ be languages such that

$F_{\omega \omega}$ is the first order language of $F$,

$F_{\infty}$ is the language of $F$ such that its formulas contain finitely many quantifiers and can be infinitely long,

$F_{\omega 0}$, resp. $F_{\infty 0}$, is the language consisting of those formulas of $F_{\omega \omega}$, resp. $F_{\infty \omega}$, in which no quantifiers occur,

$F_{\infty}$ is the language which we get by adding infinitely many quantifiers to $F_{\infty}$.

Let $\alpha \in F_{\infty 0}$ and $\kappa \in \operatorname{Ord}$ be such that the set $\left\{x_{\nu}, \nu<\kappa\right\}$ is the set of all variables of $\alpha$. Then we say that $\alpha$ is $\kappa$-ary. Let $\mathcal{A}=(\mathcal{A}, \mathcal{F})$ be an algebra, $a_{\nu} \in A$ for $\nu<\kappa$. The symbols

$$
\mathcal{A} \vDash \alpha\left(a_{\nu}, \nu<\kappa\right)
$$

mean that the formula $\alpha$ is true for $a_{\nu}, \nu<\kappa$ in the algebra $\mathcal{A}$.

A sentence is a formula without free variables. 
Let $\Sigma$ be a set of sentences of $F_{\infty}$. We denote by $\Sigma^{*}$ the class of all algebras of type $F$ which satisfy all sentences of $\Sigma$. The class $\Sigma^{*}$ is called axiomatic, cf.[1], p.256. If $\Sigma$ consists of one sentence only, then $\Sigma^{*}$ called elementary.

Let $\mathcal{K}$ be a class of algebras of type $F$. The symbol $\mathcal{K}^{*}$ denotes the class of all sentences of $F_{\infty \infty}$ which are true throughout $\mathcal{K}$.

It is obvious that

LEMMA 3. $\mathbf{I}\left(\Sigma^{*}\right)=\Sigma^{*}$.

Lemma 4. Let $n \in \mathbb{N}$ and $\alpha$ be an n-ary formula of $F_{\omega 0}$, and assume that (1) holds. If $u_{0}, \ldots, u_{n-1} \in \bar{A}$ are such that

$$
\overline{\mathcal{A}} \vDash \alpha\left(u_{0}, \ldots, u_{n-1}\right),
$$

then there are $p \in P$ and $a_{0}, \ldots, a_{n-1} \in A_{p}$ such that

$$
\mathcal{A}_{p} \vDash \alpha\left(a_{0}, \ldots, a_{n-1}\right) \text { and } a_{0} \in u_{0}, \ldots, a_{n-1} \in u_{n-1} .
$$

Proof: Let $m, i_{1}, \ldots, i_{m} \in \mathbb{N}$ and a disjunctive normal form of $\alpha$ is

$$
\bigvee_{k=1}^{m} \bigwedge_{l=1}^{i_{k}} \alpha_{k l} .
$$

Then $\alpha_{k l}$ is an equality or an unequality of two term operations of $F$ for every $k \in\{1, \ldots, m\}$ and $l \in\left\{1, \ldots, i_{k}\right\}$. In view of

$$
\overline{\mathcal{A}} \vDash \alpha\left(u_{0}, \ldots, u_{n-1}\right)
$$

there exists $k \in\{1, \ldots, m\}$ such that

$$
\overline{\mathcal{A}} \vDash \alpha_{k l}\left(u_{0}, \ldots, u_{n-1}\right) \text { for every } l \in\left\{1, \ldots, i_{k}\right\} .
$$

Consider $s \in P$ and $a_{0} \in u_{0} \cap A_{s}, \ldots, a_{n-1} \in u_{n-1} \cap A_{s}$. If $\alpha_{k l}$ is an unequality, then by the direct limit construction

$$
\mathcal{A}_{s} \vDash \alpha_{k l}\left(a_{0}, \ldots, a_{n-1}\right) .
$$

Therefore if $\alpha_{k l}$ is an unequality for every $l \in\left\{1, \ldots, i_{k}\right\}$, then

$$
\mathcal{A}_{s} \vDash \alpha\left(a_{0}, \ldots, a_{n-1}\right) .
$$

Suppose that $j \in\left\{1, \ldots, i_{k}\right\}$ is such that $\alpha_{k 1}, \ldots, \alpha_{k j}$ are equalities and $\alpha_{k j+1}, \ldots, \alpha_{k i_{k}}$ are unequalities. Let $l \in\{1, \ldots, j\}$. Then there exists $p_{l} \in P$ and $a_{l 0}, \ldots, a_{l n-1} \in A_{p_{l}}$ such that 


$$
a_{l 0} \in u_{0}, \ldots, a_{l n-1} \in u_{n-1} \text { and } \mathcal{A}_{p_{l}} \vDash \alpha_{k l}\left(a_{l 0}, \ldots, a_{l n-1}\right)
$$

according to Lemma 2. In view of the direct limit definition take $p \in P$ such that $p \geq p_{l}$ for all $l \in\{1, \ldots, j\}$ and $\varphi_{p_{l} p}\left(a_{l t}\right)=\varphi_{p_{l^{\prime}} p}\left(a_{l^{\prime} t}\right)$ for $l, l^{\prime} \in$ $\{1, \ldots, j\}, t \in\{0, \ldots, n-1\}$. Denote

$$
a_{0}=\varphi_{p_{1} p}\left(a_{10}\right), \ldots, a_{n-1}=\varphi_{p_{1} p}\left(a_{1 n-1}\right) .
$$

The assertion of the lemma is valid for $a_{0}, \ldots, a_{n-1}$.

We say that a formula $\alpha$ is positive, if it is build up from atomic formulas using only finite or infinite connectives $\vee, \wedge$.

Lemma 5. Let $\kappa \in$ Ord and $\alpha$ be a $\kappa$-ary positive formula of $F_{\infty 0}$. Let (1) be valid. Let $p, q \in P, p \leq q, a_{\nu} \in A_{p}$ for every $\nu<\kappa$. If

$$
\mathcal{A}_{p} \vDash \alpha\left(a_{\nu} ; \nu<\kappa\right),
$$

then

$$
\overline{\mathcal{A}} \vDash \alpha\left(\overline{a_{\nu}} ; \nu<\kappa\right) \text { and } \mathcal{A}_{q} \vDash \alpha\left(\varphi_{p q}\left(a_{\nu}\right) ; \nu<\kappa\right) \text {. }
$$

Proof: Let $f, g \in T_{F}$ be such that the formula

$$
f\left(x_{i_{1}}, \ldots, x_{i_{n}}\right)=g\left(x_{j_{1}}, \ldots, x_{j_{m}}\right),
$$

is contained in $\alpha, m, n \in \mathbb{N}_{0}, i_{1}<\ldots<i_{n}<\kappa, j_{1}<\ldots<j_{m}<\kappa$. Suppose

$$
f\left(a_{i_{1}}, \ldots, a_{i_{n}}\right)=g\left(a_{j_{1}}, \ldots, a_{j_{m}}\right) .
$$

The direct limit definition gives

$$
f\left(\overline{a_{i_{1}}}, \ldots, \overline{a_{i_{n}}}\right)=g\left(\overline{a_{j_{1}}}, \ldots, \overline{a_{j_{m}}}\right)
$$

and for every $q \in P, q \geq p$

$$
f\left(\varphi_{p q}\left(a_{i_{1}}\right), \ldots, \varphi_{p q}\left(a_{i_{n}}\right)\right)=g\left(\varphi_{p q}\left(a_{j_{1}}\right), \ldots, \varphi_{p q}\left(a_{j_{m}}\right)\right) .
$$

Therefore the assertion of the lemma is valid, since $\alpha$ is positive.

We denote by $\Gamma$ the class of all sentences $\gamma$ of $F_{\infty}$ such that $\gamma$ is equivalent to a sentence of the form

$$
\left(\forall x_{0}, \ldots, x_{n-1}\right)\left(\alpha \rightarrow\left(\exists y_{\mu} ; \mu<\varrho\right) \beta\right),
$$

where $\alpha$ is a formula of $F_{\omega 0}, \beta$ is a positive formula of $F_{\infty 0}, n \in \mathbb{N}_{0}$ and $\varrho \in$ Ord. 
TheOrem 1. If $\gamma \in \Gamma$, then $\{\gamma\}^{*}$ is an $\underline{\mathbf{L}}$-class.

Proof: Suppose that $\gamma$ is a sentence

$$
\left(\forall x_{0}, \ldots, x_{n-1}\right)\left(\alpha \rightarrow\left(\exists y_{\mu} ; \mu<\varrho\right) \beta\right),
$$

where $\alpha$ is a formula of $F_{\omega 0}, \beta$ is a positive formula of $F_{\infty 0}, n \in \mathbb{N}$ and $\varrho$ is an ordinal number. Denote $\mathcal{K}=\{\gamma\}^{*}$.

Let (1) be valid and $\mathcal{A}_{p} \in \mathcal{K}$ for every $p \in P$.

Suppose that $n>0$ and $u_{0}, \ldots, u_{n-1} \in \bar{A}$ are such that

$$
\overline{\mathcal{A}} \vDash \alpha\left(u_{0}, \ldots, u_{n-1}\right) .
$$

Therefore there exist $p \in P$ and $a_{0}, \ldots, a_{n-1} \in A_{p}$ such that

$$
a_{0} \in u_{0}, \ldots, a_{n-1} \in u_{n-1} \text { and } \mathcal{A}_{p} \vDash \alpha\left(a_{0}, \ldots, a_{n-1}\right)
$$

according to the Lemma 4 .

Since $\mathcal{A}_{p} \in \mathcal{K}$ we get that there exist $b_{\mu} \in A_{p}, \mu<\varrho$ such that $\mathcal{A}_{p} \vDash$ $\beta\left(a_{0}, \ldots, a_{n-1}, b_{\mu} ; \mu<\varrho\right)$. We obtain that

$$
\overline{\mathcal{A}} \vDash \beta\left(u_{0}, \ldots, u_{n-1}, \overline{b_{\mu}} ; \mu<\varrho\right)
$$

according to Lemma 5 . Therefore

$$
\overline{\mathcal{A}} \vDash \gamma\left(u_{0}, \ldots, u_{n-1}, \overline{b_{\mu}} ; \mu<\varrho\right) .
$$

Conclude that $\overline{\mathcal{A}} \in \mathcal{K}$.

If $n=0, \varrho>0$, then we take in the proof above arbitrary $p \in P$.

If $n=0, \varrho=0$, then it follows from the direct limit definition.

Corollary 1. Let $\mathcal{K}$ be a class of algebras of type $F$. Then

$$
\underline{\mathbf{L}} \mathcal{K} \subseteq\left(\mathcal{K}^{*} \cap \Gamma\right)^{*}
$$

Proof: Let (1) be valid and $\mathcal{A}_{p} \in \mathcal{K}$. Take $\gamma \in \mathcal{K}^{*} \cap \Gamma$. Then $\gamma$ is valid in $\mathcal{A}_{p}$. Thus $\gamma$ is valid in $\overline{\mathcal{A}}$ according to the Theorem 1 .

\section{The first order case}

Let $\Sigma$ be a set of sentences of $F_{\omega \omega}$. We will see in this section that direct limits built on natural numbers with linear order decide if the class $\Sigma^{*}$ is direct limit closed. We use the paper [8] of H.J.Keisler to obtain a characterization of all axiomatic $\underline{\mathbf{L}}$-classes over $F_{\omega \omega}$. 
Let $\mathcal{B}=(B, F)$. Let $\mathbf{S u b}(\mathcal{B})$ be the set of all algebras $\mathcal{A}=(A, F)$ such that

(i) $A \subseteq B$,

(ii) for any $n$-ary formula $\alpha$ of $F_{\omega \omega}$ and $a_{1}, \ldots, a_{n} \in A$ there is

$$
\mathcal{A} \vDash \alpha\left(a_{1}, \ldots, a_{n}\right) \text { if and only if } \mathcal{B} \vDash \alpha\left(a_{1}, \ldots, a_{n}\right) \text {. }
$$

Let $\mathcal{K}$ be a class of algebras of type $F$. We denote

$$
\operatorname{Sub}(\mathcal{K})=\bigcup_{\mathcal{B} \in \mathcal{K}} \operatorname{Sub}(\mathcal{B})
$$

Following two lemmas follow immediately from definition.

Lemma 6. Let $\Sigma$ be a set of sentences. Then $\operatorname{Sub}\left(\Sigma^{*}\right)=\Sigma^{*}$.

Lemma 7. Let $\mathcal{K}$ be a class of algebras of type $F$. Then

$$
\operatorname{Sub}(\mathbf{I}(\mathcal{K}))=\mathbf{I}(\operatorname{Sub}(\mathcal{K}))
$$

We denote by $\Delta$ be the class of all sentences $\gamma$ of $F_{\omega \omega}$ such that $\gamma$ is equivalent to a sentence of the form

$$
\bigwedge_{i=1}^{m}\left(\forall x_{1}, \ldots, x_{s}\right)\left(\alpha_{i} \rightarrow\left(\exists x_{s+1}, \ldots, x_{t}\right) \beta_{i}\right),
$$

where $m \in \mathbb{N}, \alpha_{i}, \beta_{i}$ are positive formulas of $F_{\omega 0}$ for every $i=1, \ldots, m$, $s, t \in \mathbb{N}_{0}, s \leq t$.

LEMMA 8.

$$
\Delta \subset\left\{\bigwedge_{i=1}^{m} \delta_{i}: m \in \mathbb{N}, \delta_{i} \in \Gamma \text { for each } i=1, \ldots, m\right\} .
$$

Let (1) be valid and let $\langle P, \leq\rangle$ be the set of positive integers with usual linear order, $\mathcal{A}_{p} \in \mathcal{K}$. Then we say that $\overline{\mathcal{A}} \in \operatorname{DirLim}(\mathcal{K})$.

The following statement is an immediate consequence of Theorem 6 of [8]. The proof uses so called "method of diagrams". This method requires to change the type of algebras by adding an (infinite) set of nullary operations to $F$. 
TheOREm 2. Let $\Sigma$ be a set of sentences of $F_{\omega \omega}$ and $\mathcal{K}=\Sigma^{*}$. Then

$$
\operatorname{Sub}(\operatorname{DirLim} \mathcal{K})=\left(\mathcal{K}^{*} \cap \Delta\right)^{*} .
$$

The class $\operatorname{Sub}(\mathcal{K})$ is denoted by $F_{\omega \omega}-\operatorname{Sub}(\mathcal{K})$ in [8]. Let $F^{\prime}$ be the least generalized atomic set of formulas from $F_{\omega \omega}$ such that the $(n+1)$ ary formula $f\left(x_{0}, \ldots, x_{n-1}\right)=x_{n}$ belongs to $F^{\prime}$ for every $n$-ary operation $f \in F, n \in \mathbb{N}$. The class $\operatorname{DirLim}(\mathcal{K})$ is denoted by $F^{\prime}-\operatorname{DirLim}(\mathcal{K})$ and the set $\Delta=\forall \mathbf{B} \exists \vee \wedge F^{\prime}$ in [8].

TheOREM 3. Let $\Sigma$ be a set of sentences of $F_{\omega \omega}$ and $\mathcal{K}=\Sigma^{*}$. The following statements are equivalent

(i) $\mathcal{K}$ is an $\underline{\mathbf{L}}$-class,

(ii) $\mathbf{I}(\underline{\mathbf{L}}(\mathcal{K}))=\mathcal{K}$,

(iii) $\mathbf{I}(\operatorname{DirLim}(\mathcal{K}))=\mathcal{K}$,

(iv) $\mathcal{K}=\left(\mathcal{K}^{*} \cap \Delta\right)^{*}$.

Proof: Statements (i) and (ii) are equivalent according to $\mathbf{I}(\mathcal{K})=\mathcal{K}$.

Assume that (ii) is valid. We have $\operatorname{DirLim}(\mathcal{K}) \subseteq \underline{\mathbf{L}}(\mathcal{K})$. Therefore $\mathbf{I}(\operatorname{DirLim}(\mathcal{K})) \subseteq \mathbf{I}(\underline{\mathbf{L}}(\mathcal{K}))$. Thus $\mathbf{I}(\operatorname{DirLim}(\mathcal{K})) \subseteq \mathcal{K}$. The opposite inclusion is obvious according to Lemma 1.

Assume that (iii) is valid. We observe

$$
\operatorname{Sub}(\mathbf{I}(\operatorname{DirLim}(\mathcal{K})))=\mathbf{I}(\operatorname{Sub}(\operatorname{DirLim}(\mathcal{K})))=\mathbf{I}\left(\left(\mathcal{K}^{*} \cap \Delta\right)^{*}\right)=\left(\mathcal{K}^{*} \cap \Delta\right)^{*}
$$

in view of Lemma 7, Theorem 2 and Lemma 3. We obtain

$$
\mathcal{K}=\operatorname{Sub}(\mathcal{K})=\operatorname{Sub}(\mathbf{I}(\operatorname{Dir} \operatorname{Lim}(\mathcal{K})))=\left(\mathcal{K}^{*} \cap \Delta\right)^{*}
$$

according to Lemma 6 .

Assume that (iv) is valid. Let $\mathcal{K}=\left\{\gamma_{r}, r \in R\right\}^{*}$ and $\gamma_{r} \in \Delta$ for each $r \in R$. Then $\mathcal{K}=\bigcap_{r \in R}\left\{\gamma_{r}\right\}^{*}$. Let $r \in R$. In view of Lemma 8 we have that $\gamma_{r}=\bigwedge_{i=1}^{m_{r}} \delta_{r i}$ for some $m_{r} \in \mathbb{N}, \delta_{i r} \in \Gamma$ for each $i=1, \ldots, m_{r}$. We get

$$
\mathcal{K}=\bigcap_{r \in R} \bigcap_{i=1}^{m_{r}}\left\{\delta_{r i}\right\}^{*} .
$$

The class $\left\{\delta_{r i}\right\}^{*}$ is an $\underline{\mathbf{L}}$-class according to $\delta_{r i} \in \Gamma$ and Theorem 1. Because $\underline{\mathbf{L}}$-classes are closed with respect to intersections we obtain that $\mathcal{K}$ is an $\overrightarrow{\mathbf{L}}$-class. 


\section{Connected monounary algebras}

The rest of this paper we will deal with monounary algebras. We suppose that $h$ is an unary operational symbol and $F=\{h\}$. We will write $\mathcal{A}=$ $(A, h)$ instead of $\mathcal{A}=(A,\{h\})$. For monounary terminology and basic properties see e.g. [7], [11].

We will see that the class of all connected monounary algebras is an axiomatic class which is direct limit closed and it is not an axiomatic class of the first order logic in this section.

Lemma 9. The class of all connected monounary algebras is an axiomatic $\underline{\mathrm{L}}$-class.

Proof: Let $\gamma$ be a sentence

$$
(\forall x, y, z)\left(z=z \rightarrow \bigvee_{m, n \in \mathbb{N}} h^{m}(x)=h^{n}(y)\right) .
$$

Then $\gamma \in F_{\infty}$ and $\{\gamma\}^{*}$ is the class of all connected monounary algebras. Since $\gamma \in \Gamma$, the class $\{\gamma\}^{*}$ is an $\underline{\mathbf{L}}$-class according to Theorem 1 .

LEMMA 10. The class of all connected monounary algebras is not closed with respect to ultraproducts.

Proof: Let $\mathcal{F}$ be an ultrafilter which contains $\{J \subseteq \mathbb{N}: \mathbb{N}-J$ is finite $\}$. Let $\left(A_{i}, h\right)$ be isomorphic to a ray, i.e. natural numbers with a successor operation, for every $i \in \mathbb{N}$. Consider the ultraproduct

$$
\prod_{i \in \mathbb{N}}\left(A_{i}, h\right) / \mathcal{F} .
$$

Let $a=(1,1,1, \ldots), b=(1,2,3, \ldots)$. We have $\left\{i \in \mathbb{N}: a_{i}=b_{i}\right\}=\{1\} \notin \mathcal{F}$ in view of $\mathbb{N}-\{1\} \in \mathcal{F}$. Thus $a$ and $b$ are different elements of $\prod_{i \in \mathbb{N}} A_{i} / \mathcal{F}$.

Suppose that $h^{n}(a)=h^{m}(b)$ for some $m, n \in \mathbb{N}_{0}$ in the algebra $\prod_{i \in \mathbb{N}}\left(A_{i}, h\right) / \mathcal{F}$. We have $h^{n}(a)=(n+1, n+1, n+1, \ldots), h^{m}(b)=(m+1$, $m+2, m+3, \ldots)$ by the definition. Therefore there exists $k \in \mathbb{N}_{0}$ such that $m=n+k$ or $n=m+k$. If $n=m+k$, then $\{i \in \mathbb{N}: m+$ $k=m+(i-1)\}=\{k+1\} \notin \mathcal{F}$. If $k>0$ and $m=n+k$, then $\{i \in \mathbb{N}: n=n+k+(i-1)\}=\emptyset$. Conclude that $a, b$ are in different components of the algebra $\prod_{i \in \mathbb{N}}\left(A_{i}, h\right) / \mathcal{F}$. 
Corollary 2. Let $\Sigma$ be a set of sentences of $F_{\omega \omega}$. Then $\Sigma^{*}$ is not a class of all connected monounary algebras.

Proof: It follows from the previous lemma and Corollary 9.5.10 of [4].

\section{Monounary sentences preserved by direct limits}

In this section we will prove that Theorem 1 can be extended in monounary algebras . Namely the formula $\alpha$ in the sentence $\gamma$ can be considered infinite with finitely many variables.

We remind that a finite connected monounary algebra with an injective operation is a cycle called. The length of a cycle is the number of elements of this cycle.

Let $d \in \mathbb{N}, r \in \mathbb{N}_{0}$. Let $\mathcal{A}=(A, h)$ be a monounary algebra. Suppose that $a \in A$ and $C \subseteq A$ are such that

1) $(C, h)$ is a cycle of length $d$,

2) $h^{r}(a) \in C$,

3) if $r>0$, then $h^{r-1}(a) \notin C$.

Then we will say that $a$ has $(d, r)$-property in the algebra $\mathcal{A}$.

The following two facts will be used:

Lemma 11. Let $\mathcal{A}=(A, h)$ be a connected monounary algebra with a cycle of length $d, a, b \in A$. Then a has $(d, r)$-property and $b$ has $\left(d, r^{\prime}\right)$-property for some $r, r^{\prime} \in \mathbb{N}_{0}$.

Lemma 12. Let $g \in T_{F}$ be $n$-ary, $n \in \mathbb{N}$. Then there exists $m \in \mathbb{N}_{0}$ and $i \in\{0,1, \ldots, n-1\}$ such that $g\left(x_{0}, \ldots, x_{n-1}\right)=h^{m}\left(x_{i}\right)$.

Lemma 13. Let $\mathcal{A}=(A, h)$ be a connected monounary algebra with a cycle of length $d, a, b \in A$. Let $r, r^{\prime} \in \mathbb{N}_{0}$ be such that a has $(d, r)$-property and $b$ has $\left(d, r^{\prime}\right)$-property. Let $q, q^{\prime}, t, t^{\prime} \in \mathbb{N}_{0}$ be such that $t \leq t^{\prime}<d$, $k=r+q d+t, l=r^{\prime}+q^{\prime} d+t^{\prime}$. Then the following statements are equivalent

(a) $h^{k}(a)=h^{l}(b)$

(b) $h^{r+t}(a)=h^{r^{\prime}+t^{\prime}}(b)$,

(c) $h^{r}(a)=h^{r^{\prime}+\left(t^{\prime}-t\right)}(b)$,

(d) $h^{r+d-\left(t^{\prime}-t\right)}(a)=h^{r^{\prime}}(b)$. 
Proof: The equality (b) follows from (a) because $h^{r}(a), h^{r^{\prime}}(b)$ are cyclic elements. The statement (c) results from (b) because any cycle operation is injective. It is obvious that (c) implies (b).

Let (b) be valid. Then

$$
\begin{gathered}
h^{k}(a)=h^{r+q d+t}(a)=h^{q d+t}\left(h^{r}(a)\right)=h^{t}\left(h^{r}(a)\right)=h^{t+r}(a)= \\
=h^{r^{\prime}+t^{\prime}}(b)=h^{t^{\prime}}\left(h^{r^{\prime}}(b)\right)=h^{q^{\prime} d+t^{\prime}}\left(h^{r^{\prime}}(b)\right)=h^{r^{\prime}+q^{\prime} d+t^{\prime}}(b)=h^{l}(b)
\end{gathered}
$$

since $h^{r}(a), h^{r^{\prime}}(b)$ are elements of the cycle of length $d$.

If $c, e$ are elements of one cycle of length $d$ and $h^{n}(c)=e$, then $h^{d-n}(e)=c$. This property gives that (c) and (d) are equivalent.

Lemma 14. Let (1) be valid, $p \in P$ and $C$ be a cycle of the algebra $\mathcal{A}_{p}$. Then $\bar{C}=\{\bar{c}: c \in C\}$ is a cycle of the algebra $\overline{\mathcal{A}}$ and $|\bar{C}| /|C|$.

Proof: Let $\varphi$ be a mapping from $A_{p}$ into $\bar{A}$ such that $\varphi(a)=\bar{a}$ for every $a \in A_{p}$. Then $\varphi$ is a homomorphism from $\mathcal{A}_{p}$ into $\overline{\mathcal{A}}$.

Lemma 15. Let (1) be valid and $d \in \mathbb{N}, r \in \mathbb{N}_{0}$.

If $u \in \bar{A}$ has $(d, r)$-property in $\overline{\mathcal{A}}$, then there exist $p \in P$ and $a \in A_{p}$ such that a has $(d, r)$-property in $\mathcal{A}_{p}$.

Proof: Take $b \in u$. Then $b \in A_{s}$ for some $s \in P$. We obtain

$$
\overline{h^{r}(b)}=h^{r}(\bar{b})=h^{r}(u)=h^{r+d}(u)=h^{r+d}(\bar{b})=\overline{h^{r+d}(b)} .
$$

Thus there exists $p \in P, p \geq s$ such that

$$
\varphi_{s p}\left(h^{r}(b)\right)=\varphi_{s p}\left(h^{r+d}(b)\right) .
$$

Therefore

$$
h^{r}\left(\varphi_{s p}(b)\right)=h^{r+d}\left(\varphi_{s p}(b)\right) .
$$

Denote $a=\varphi_{s p}(b)$. Let $C$ be a cycle of $\mathcal{A}_{p}$ such that $h^{r}(a) \in C$. We have $|C|=d$ according to the previous lemma. Assume that $h^{r-1}(a) \in C$. Then $h^{r-1}(u)=h^{r-1}(\bar{a})=\overline{h^{r-1}(a)}$ is a cyclic element of $\overline{\mathcal{A}}$ according to the previous lemma, a contradiction. 
Corollary 3. Let $n \in \mathbb{N}_{0}$ and $\delta$ be n-ary formula of $F_{\omega 0}$. Let (1) be valid. If $u_{0}, \ldots, u_{n-1} \in \bar{A}$ are such that

$$
\overline{\mathcal{A}} \vDash \alpha\left(u_{0}, \ldots, u_{n-1}\right),
$$

then there are $p \in P$ and $a_{0}, \ldots, a_{n-1} \in A_{p}$ such that

(i) $a_{0} \in u_{0}, \ldots, a_{n-1} \in u_{n-1}$,

(ii) $\mathcal{A}_{p} \vDash \delta\left(a_{0}, \ldots, a_{n-1}\right)$,

(iii) if $d \in \mathbb{N}, r \in \mathbb{N}_{0}$ and $i \in\{0, \ldots, n-1\}$ are such that $u_{i}$ has $(d, r)$ property in $\overline{\mathcal{A}}$, then $a_{i}$ has $(d, r)$-property in $\mathcal{A}_{p}$.

Theorem 4. Let $h$ be an unary operational symbol and $F=\{h\}$. Let $\alpha$, $\beta$ be formulas of $F_{\infty 0}, \beta$ be positive, $n \in \mathbb{N}$ and $\varrho$ be an ordinal number. If $\gamma$ is a sentence

$$
\left(\forall x_{0}, \ldots, x_{n-1}\right)\left(\alpha \rightarrow\left(\exists y_{\mu} ; \mu<\varrho\right) \beta\right),
$$

then $\{\gamma\}^{*}$ is an $\underline{\mathbf{L}}$-class.

Proof: Denote $\mathcal{K}=\{\gamma\}^{*}$.

Let (1) be valid and $\mathcal{A}_{p} \in \mathcal{K}$ for every $p \in P$.

Suppose that $u_{0}, \ldots, u_{n-1} \in \bar{A}$ are such that $\overline{\mathcal{A}} \vDash \alpha\left(u_{0}, \ldots, u_{n-1}\right)$. Let $i \in\{0, \ldots, n-1\}$. If a component which contains $u_{i}$ has a cycle, then let $d_{i} \in \mathbb{N}, r_{i} \in \mathbb{N}_{0}$ be such that $u_{i}$ has $\left(d_{i}, r_{i}\right)$-property in $\overline{\mathcal{A}}$. We have that $u_{i}$ is cyclic or $u_{i}$ is not cyclic in $\overline{\mathcal{A}}$. If $u_{i}$ is cyclic, then $r_{i}=0$. If $u_{i}$ is not cyclic, then $r_{i}$ is not defined or $r_{i}>0$. Without loss of generality let $m \in \mathbb{N}_{0}$ be such that $u_{0}, \ldots, u_{m-1}$ are not cyclic and $u_{m}, \ldots, u_{n-1}$ are cyclic elements of $\overline{\mathcal{A}}$. Put

$$
M=\left\{(i, j): i<j \text { and } u_{i}, u_{j} \text { are in the same component of } \overline{\mathcal{A}}\right\} .
$$

Then $M$ is finite. Let

$$
\begin{gathered}
M_{1}=\left\{(i, j) \in M: \text { there exist } k, l \in \mathbb{N}_{0} \text { such that } h^{k}\left(u_{i}\right)=h^{l}\left(u_{j}\right)\right. \\
\text { and } \left.h^{k}\left(u_{i}\right) \text { is not cyclic }\right\} .
\end{gathered}
$$

For every $(i, j) \in M_{1}$ let $k_{i j}, l_{i j}$ be such that $h^{k_{i j}}\left(u_{i}\right)=h^{l_{i j}}\left(u_{j}\right), h^{k_{i j}}\left(u_{i}\right)$ is not cyclic and $h^{k_{i j}-1}\left(u_{i}\right) \neq h^{l_{i j}-1}\left(u_{j}\right)$. For every $(i, j) \in M-M_{1}$ let $l_{i j}=r_{j}$ and $k_{i j}$ be the least possible such that $h^{k_{i j}}\left(u_{i}\right)=h^{l_{i j}}\left(u_{j}\right)$. 
Let $\delta$ be a formula

$$
\bigwedge_{(i, j) \in M} h^{k_{i j}}\left(x_{i}\right)=h^{l_{i j}}\left(x_{j}\right) .
$$

Then $\delta$ is from $F_{\omega 0}$ and

$$
\overline{\mathcal{A}} \vDash \delta\left(u_{0}, \ldots, u_{n-1}\right) .
$$

Take $p \in P$ and $a_{0}, \ldots, a_{n-1} \in A_{p}$ which satisfy conditions (i)-(iii) from Corollary 3.

Let a disjunctive normal form of $\alpha$ be

$$
\bigvee_{s \in S} \bigwedge_{t \in T_{s}} \alpha_{s t}
$$

Since $\overline{\mathcal{A}} \vDash \alpha\left(u_{0}, \ldots, u_{n-1}\right)$ we have that there is $s \in S$ such that $\overline{\mathcal{A}} \vDash \alpha_{s t}\left(u_{0}, \ldots, u_{n-1}\right)$ for every $t \in T_{s}$.

Consider $t \in T_{s}$. The formula $\alpha_{s t}$ is an equality or an unequality of two term operations of $F$. One or two variables occur in $\alpha_{s t}$ according to Lemma 12. Let $x_{i}, x_{j}$ be variables of $\alpha_{s t}, i, j \in\{0, \ldots, n-1\}$.

If $\alpha_{s t}$ is an unequality, then for every $q \in P$ and $b_{i} \in u_{i} \cap A_{q}, b_{j} \in$ $u_{j} \cap A_{q}$ we have that $\mathcal{A}_{q} \vDash \alpha_{s t}\left(b_{i}, b_{j}\right)$ according to the direct limit definition. Therefore $\mathcal{A}_{p} \vDash \alpha_{s t}\left(a_{i}, a_{j}\right)$.

Let $\alpha_{s t}$ be an equality. Then $\alpha_{s t}$ is $h^{k}\left(x_{i}\right)=h^{l}\left(x_{j}\right)$ for some $k, l \in \mathbb{N}_{0}$.

Let $i=j$ and $k>l$. Then $h^{k}\left(u_{i}\right)$ is cyclic and $k, l \geq r_{i}$. Further, $k-l=q d_{i}$ for some $q \in \mathbb{N}$. We obtain

$$
h^{k}\left(a_{i}\right)=h^{l+k-l}\left(a_{i}\right)=h^{k-l}\left(h^{l}\left(a_{j}\right)\right)=h^{q d}\left(h^{l}\left(a_{j}\right)\right)=h^{l}\left(a_{j}\right),
$$

since $h^{l}\left(a_{j}\right)$ is cyclic in $\mathcal{A}_{p}$ according to Corollary 3(iii). Therefore $\mathcal{A}_{p} \vDash$ $\alpha_{s t}\left(a_{i}, a_{j}\right)$.

Suppose that $i<j$. Then $(i, j) \in M$. Let $(i, j) \in M_{1}$. If $h^{k}\left(u_{i}\right)$ is not cyclic, then $k=k_{i j}+t$ and $l=l_{i j}+t$ for some $t \in N_{0}$. We obtain

$$
h^{k}\left(a_{i}\right)=h^{k_{i j}+t}\left(a_{i}\right)=h^{t}\left(h^{l_{i j}}\left(a_{j}\right)\right)=h^{l}\left(a_{j}\right) .
$$

If $h^{k}\left(u_{i}\right)$ is cyclic, then two cases can occur. The first one is that $k=$ $k_{i j}+t+q d, l=l_{i j}+t$ for some $t \in \mathbb{N}, d \in \mathbb{N}_{0}$. Then

$$
h^{k}\left(a_{i}\right)=h^{k_{i j}+t+q d}\left(a_{i}\right)=h^{t+q d}\left(h^{l_{i j}}\left(a_{j}\right)\right)=h^{q d}\left(h^{l}\left(a_{j}\right)\right)=h^{l}\left(a_{j}\right)
$$

since $h^{l}\left(a_{j}\right)$ belongs to a cycle of length $d$ according Corollary 3(iii). The second case is that $k=k_{i j}+t, l=l_{i j}+t+q d$ for some $t \in \mathbb{N}, d \in \mathbb{N}_{0}$. Then analogously as above we have that $h^{l}\left(a_{j}\right)=h^{k}\left(a_{i}\right)$. 
Let $(i, j) \notin M_{1}$. Denote $d=d_{i}\left(=d_{j}\right)$. Consider $t, t^{\prime}, q, q^{\prime} \in \mathbb{N}_{0}$ such that $t, t^{\prime}<d$ and $k=r_{i}+q d+t, l=r_{j}+q^{\prime} d+t^{\prime}$.

Assume that $t \leq t^{\prime}$. Then $h^{r_{i}+d-\left(t^{\prime}-t\right)}\left(u_{i}\right)=h^{r_{j}}\left(u_{j}\right)$ according to Lemma $13(\mathrm{~d})$. Since $l_{i j}=r_{j}$, we obtain that $k_{i j}=r_{i}+d-\left(t^{\prime}-t\right)$. That means $h^{k}\left(a_{i}\right)=h^{l}\left(a_{j}\right)$ in view of $h^{k_{i j}}\left(a_{i}\right)=h^{l_{i j}}\left(a_{j}\right)$, Corollary 3(iii) and Lemma 13(a).

Assume that $t^{\prime}<t$. Then $h^{r_{j}}\left(u_{j}\right)=h^{r_{i}+\left(t-t^{\prime}\right)}\left(u_{i}\right)$ according to Lemma 13(c). Since $l_{i j}=r_{j}$, we obtain that $k_{i j}=r_{i}+\left(t^{\prime}-t\right)$. That means $h^{k}\left(a_{i}\right)=$ $h^{l}\left(a_{j}\right)$ in view of $h^{k_{i j}}\left(a_{i}\right)=h^{l_{i j}}\left(a_{j}\right)$, Corollary 3(iii) and Lemma 13(a).

Conclude that $\mathcal{A}_{p} \vDash \alpha\left(a_{0}, \ldots, a_{n-1}\right)$.

Since $\mathcal{A}_{p} \in \mathcal{K}$ we get that there exist $b_{\mu} \in A_{p}, \mu<\varrho$ such that $\mathcal{A}_{p} \vDash$ $\beta\left(a_{0}, \ldots, a_{n-1}, b_{\mu} ; \mu<\varrho\right)$. We obtain that $\overline{\mathcal{A}} \in \mathcal{K}$ analogously as in the proof of Theorem 1 .

\section{Conclusions and open problems}

1. Theorem 3 says that every axiomatic class determined by some firstorder sentences is closed with respect to direct limits if and only if it is an axiomatic class determined by some sentences from $\Delta$. The question, whether an analogous statement about axiomatic classes generally and the class of sentences $\Gamma$ is valid, is open. It is interesting even in the case of elementary classes.

2. Theorem 1 states that the elementary class of each sentence of $\Gamma$ is the class of algebras closed under direct limits. We can see in Theorem 4 that Theorem 1 can be strengthened in the case of monounary algebras by allowing the formula $\alpha$ to be a formula of infinite length with finitely many variables. The proof of Theorem 4 uses significantly the graph structure of monounary algebras. Are there another types of algebras allowing the same amplification? Find examples where this amplification does not work.

3. Section 5 shows the direct limit closed class of algebras which is not possible to express as an axiomatic one determined by some firstorder sentences. This class is elementary determined by a sentence from $\Gamma$. Is every direct limit closed class axiomatic? 


\section{References}

[1] G. Grätzer, Universal Algebra. The University Series in Higher Mathematics, D. Van Nostrand, Co., Princeton, N.Y., 1968.

[2] E. Halušková, Direct limits of monounary algebras, Czechoslovak Math. J. 49 (1999), pp. 645-656.

[3] E. Halušková, M. Ploščica, On direct limits of finite algebras, Contributions to general algebra 11 (1999), Heyn, Klagenfurt, pp. 101-104.

[4] W. Hodges, Model Theory. Encyclopedia of mathematics and its applications, v. 42, Cambridge University Press, 1993.

[5] T. Ihringer, Allgemeine Algebra, B. G. Teubner Stuttgart 1988.

[6] J. Jakubík, G. Pringerová, Direct limits of cyclically ordered groups, Czechoslovak Math. J. 44 (1994), pp. 231-250.

[7] D. Jakubíková-Studenovská, J. Pócs, Monounary algebras, P. J. Šafárik University in Košice, 2009.

[8] H. J. Keisler, Theory of Models with Generalized Atomic Formulas, The Journal of Symbolic Logic, Vol. 25, No. 1 (Mar.,1960), pp. 1-26.

[9] H. J. Keisler, C. C. Chang, Model Theory, Studies in Logic and the Foundation of Mathematics, vol. 73, Elsevier, 1992.

[10] C. Pelea, On the direct limit of a direct system of multialgebras, Discrete Mathematics 306 (2006), pp. 2916-2930.

[11] R. McKenzie, G. McNulty, W. Taylor, Algebras, Lattices, Varieties, vol. 1, Wadsworth, 1987.

Mathematical Institute, Slovak Academy of Sciences, Grešákova 6, 04001 Košice, Slovakia

e-mail: ehaluska@saske.sk 